reductase, because it acts on a wide range of aleohols. Lactate dehydrogenase $(E C$ 1.1.1.27) is named L-lactate : NAD oxidoreductase, even though it reacts quite rapidly with NADP as well as with NAD. However, the most commonly occurring glutamate dehydrogenase (EC 1.4.1.3) is named L-glutamate: $\mathrm{NAD}(\mathrm{P})$ oxidoreductase (deaminating), because it reacts readily with both NAD and NADP (seo Rule 16). The aldehyde dehydrogenases give special difficulties. No less than 18 are listed in Group 1.2.1 (with NAD or NADP as acceptor). Of these, 14 are named in terms of a specific hydrogen donor, while in the others the donor is given simply as aldehyde. This should not be taken to mean that the 14 are absolutely specific for a single aldehyde. Of the 18 enzymes, NAD is given as acceptor for 8, NADP for 6 and both nucleotides for 4 .
There are many discrete enzymes, differing in aminoacid composition, physical properties and enzyme kinetics, all of which have to be named aldehyde: NAD oxidoreductase ( $E C$ 1.2.1.3). At present these must be distinguished by source, such as organism, tissue and cell component. The IUB Commission of Editors of Biochemical Journals has set up a sub-committee to consider the problems of nomenclature posed by recent research on the nature of isoenzymes and enzyme sub-units.

of Biochemistry on the Nomenclature and Classification of Enzymes, together with their Units and the Symbols of Enzyme Kinetics. Pp. v+219. Report of the Commission on Enzymes of the International Union of Bio-
chemistry, 1961 (Pergamon Press, Oxford, 1961).

Biochim. Biophys. Acta, 107, 5 (1965).

\title{
INTERNATIONAL COMMITTEE ON LABORATORY ANIMALS
}

$\mathrm{T}$ HE International Committee on Laboratory Animals held its third international symposium at Dun Laoghaire, near Dublin, during Septomber 6-17 under the general title, "The Husbandry of Laboratory Animals" *. The Committee, while well known to those professionally engaged in laboratory animal science, appears to be largely unknown to the users of laboratory animals. It was, however, the users, who, acting through their international unions, set up the Committee. It seems appropriate, therefore, that a general report of the Committee and its work should be made to laboratory animal users on this occasion.

History. Ten years ago two independent initiatives towards an international organization were being made. One was by the International Union of Biological Sciences and the other by the Council for International Organizations for Medical Sciences working in association with Unesco. In December 1956 these organizations met and recommended the establishment of the International Committee on Laboratory Animals. The International Union of Physiological Sciences joined soon after, and the Committee was established as an inter-union committee; experts, who were hoads of laboratory animal centres, were co-opted. Unesco provided most of the financial support and was represented on the Committee by an observer. Two other unions have since joined-the International Union against Cancer and the International Union of Biochemistry.

The growth of the Committee's activities was such that by 1961 a revised constitution was necessary. Laboratory animal centres or committees had been established in many countries to co-ordinate work in the field and act as information centres. It seemed important to associate these bodies with the International Committee. The Committee now consists of the union members and national representatives, numbering twenty-seven, coming from all parts of the world. The final authority rests with the governing body, which consists of all the union representatives together with an equal number of national representatives; the World Health Organization is represented by an observer. In effect this is an equal division between the users and the laboratory animal oxperts. The detailed work is done by an executive committee of individual experts.

The financial support from Unesco was only temporary and in 1962 it ended. The Committee is most grateful to Unesco. The World Health Organization has now undertaken to support the work of the Committee, and to that Organization also the Committee is deeply grateful.

Activities. Surveys of the production and utilization of laboratory animals havo been carried out and published in relation to twenty-one countries. This has enabled the

* The proceedings are being published by Academio Press. problems to be assessed and has provided a strong stimulus to the development of national centres dealing with problems connected with laboratory animals. Another major factor in such dovelopment has been the visits, made under Committee auspices, of the officers and other experts to many countries to give advice on laboratory animal problems. Up to the present time this advice has been mainly concerned with the development of healthy supplies of small mammals. Similarly, other activities have been concentrated in this direction. Thus, efforts have been made to improve technician training, and notes on training courses have been published in the International Committee on Laboratory Animals Bulletin which is published twice a year. Scholarships have been awarded to a number of workers in the field, and this has enabled them to work for a time in one of the established centres, in this way helping the development of new centres elsewhere.

Two previous international symposia have been held on "Living Animal Material for Biological Research" and "The Problem of Laboratory Animal Disease". These, together with that just held, have provided a forum for the discussion of a range of scientific problems connected with the supply of healthy animals, in particular small mammals.

Besides the basic problems of improving the health, nutrition and handling of animals, two other general fields have always been on the Committee's programme. The first of these concerns more specialized questions related to the breeding of small mammals. This side of the work has included a bibliography (now taken over by the publishers of the Zeitschrift für Versuchstierkunde-. Gustav Fischer Verlag, Jena, German Democratic Republic) and the preparation of lists of agreed definitions. These are slow steps towards the difficult task of designat. ing primary type colony centres and listing the primary type colonies boing maintained. The difficulty in this task lies in specifying strains in such a way that the designations have real meaning.

The Committee is one concerned with laboratory animals of all kinds and not simply small mammals. One of its early aims was to establish world lists of sources of laboratory animals, especially lower vertebrates and invertebrates. This aim has so far proved very difficult to achieve and, with the coneontration of effort on stand. ards of care in animal houses for small mammals, little has yet been done in this field. 'This has eaused some concern, and the governing body has now put on record its hope that somo advance in the non-mammalian field may take place in the next three years.

In all these fields of activity this international organization is primarily a channel of communication between national organizations, and the latter have, as one of their functions, the giving of information not only to those 
actively involved in laboratory animal care and production, but also to any user of experimental animals. In Great Britain the centre is the Laboratory Animals Centre (director, Mr. J. Bleby), Medical Research Council Laboratories, Carshalton, Surrey, and the national representative on the International Committee is $\mathrm{Dr}$ F. J. C. Roe, Department of Experimental Pathology, Chester Beatty Research Institute, Institute of Cancer Research, Fulham Road, London, S.W.3.

\title{
BASIC COMPONENT OF SOLAR RADIO EMISSION AT CENTIMETRE AND DECIMETRE WAVE-LENGTHS
}

\author{
By DR. M. K. DAS GUPTA and D. BASU \\ Institute of Radio Physics and Electronics, University of Calcutta
}

$I^{\mathrm{T}}$ $T$ is now well established that at centi- and decimetre wave-longths the solar radio emission, apart from the more rapidly varying features like bursts of various types, consists of: (1) a basic component remaining constant ovor a considerable period of time; (2) a slowly varying componont showing a slow day-to-day variation in intensity. Of these two, the first is the radiation from the Sun when the disk is completely free from any active rogion while the slowly varying component is the excess radiation due to localized active zones on the disk. The method of separating the two components from the total solar flux was first introduced by Pawsey and Yabsley $^{1}$, who plotted the total flux against sunspot area and extrapolated the best-fitting line to the condition of zero area on the assumption that the radio flux depends on sunspots only. However, it is now confirmed ${ }^{2}$ that the regions corresponding to sunspots continue to emit radio waves in $\mathrm{cm}$ - and dm-wave-lengths for a considerable period even after the visible sunspots have disappeared and also that these start emitting much earlier than the actual appearance of the sunspots. There are thus present on the solar disk, at any instant, some active regions that are strong sources of radio emission but are not at all associated with the then visible sunspots. As such, the basic component cannot be segregated from the total flux by taking into account sunspots alone.

Attempts were made independently by Allen $^{3}$ and Covington and Harvey ${ }^{2}$ to detcrmine the basic component with this new consideration in view. However, Allen's method seems to be too complicated and does not appear to be readily applicable from a practical point of view. Regarding the analysis by Covington and Harvey, on the other hand, the assumption that the contribution from radio regions not associated with sunspots is proportional to the number of sunspot groups is only a rough approximation. We wore thus prompted to examine the aspect of the evaluation of the basic component of solar radio emission in relation to recent data.

As has already been mentioned, the slow day-to-day variations of the solar radio flux would be due to the presence of active regions, visible or invisible, on the solar disk. These variations exhibit roughly a 27 -day period of recurrence which corresponds to the apparent period of rotation of the Sun about its vertical axis. It may, however, be mentioned in this connexion that such variations in the solar radio flux oxist ${ }^{4}$ even at zero sunspot number condition which can thus be atbributed to active regions other than those associated with sunspots. It is obvious that there will be no variation at all of the daily values of solar flux if the disk is free from any active radio region, and hence, so far as the basic component is concerned, the amplitude of such variation will be zero.

In our analysis we considered solar radio flux data at frequencies $1,000,2,000,2,800,3,750$ and $9,400 \mathrm{Mc} / \mathrm{s}$. The daily values of the flux at each of these frequencies were plotted separately for the years 1958 (sunspot maximum) and 1964 (sunspot minimum). All these curves showed substantially similar variation for the individual years and indicated pronounced maxima and minima following roughly the 27-day period. Fig. 1 shows such plots for the frequency $3,750 \mathrm{Mc} / \mathrm{s}$ for the years 1958 and 1964 as well as 1961. An additional striking feature exhibited by the daily mean flux curves is that the pronounced maximum-to-minimum amplitude of this variation seems to change rather systematically with tho mean value of the flux. Thus the amplitude is highest for 1958 when the mean flux is also maximum, and it is lowest for 1964 when the mean flux is minimum, while for 1961 the condition is intermediate betwcen the two. We were thus led to examine the matter in some detail. For any frequency we determined the maximum-to-minimum amplitude of variation of the daily moan flux corresponding to each of the 27-day cycles (henceforth ealled variation amplitude or VA) individually for both the years. 'The mean values of the solar radio flux for each of the periods corresponding to these cycles were also determined. For any particular frequency and for any particular year, we thus obtained a set of VAs and the corresponding values of mean solar radio flux. These flux values were plotted against corresponding variation amplitudes. Fig. 2 shows the plots for the frequencies $1,000,2,000,3,750$ and $9,400 \mathrm{Mc} / \mathrm{s}$ separately for 1958 and for 1964 . The linear relationship between the solar flux and the amplitude of variation is at once evident. On extrapolating to zero value of VA the best-fitting line is found to cut the flux

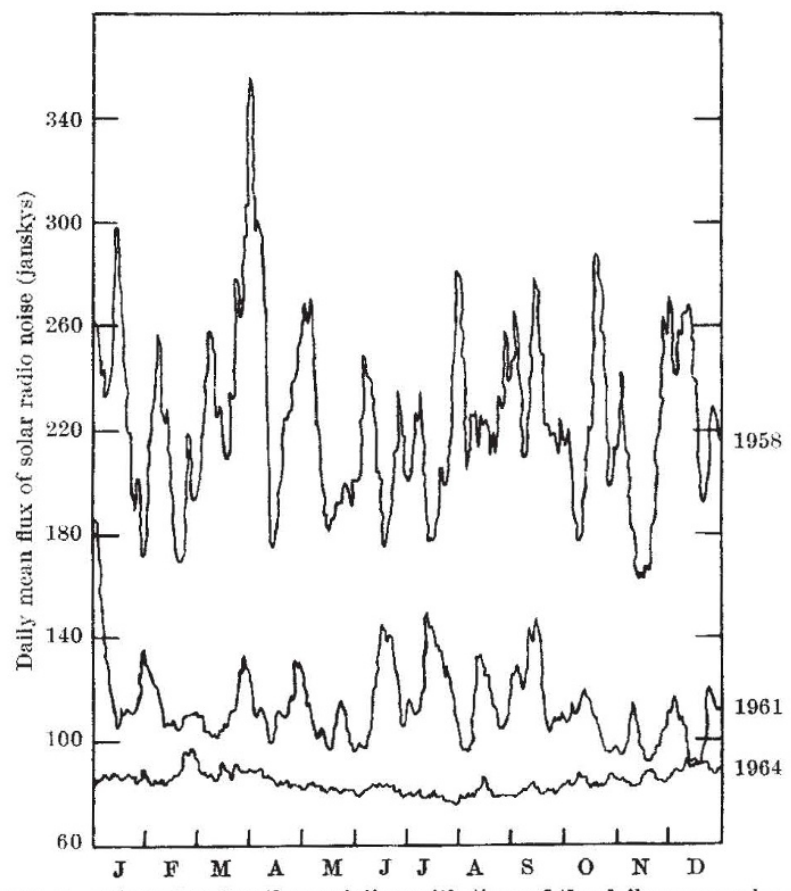

Fig. 1. Plots showing the variation with time of the daily mean solar radio flux at $3,750 \mathrm{Mc} / \mathrm{s}$ for 1958,1961 and 1964 